\title{
SUSY Enhancement of Heavy Higgs Production
}

\author{
Alexander Vadimovich Bednyakov \\ Bogoliubov Laboratory of Theoretical Physics, Joint Institute for Nuclear Research, Dubna, \\ Moscow Region, 114980, Russia \\ E-mail: bednyadtheor.jinr.ru

\section{Dmitri Igorevich Kazakov} \\ Bogoliubov Laboratory of Theoretical Physics, Joint Institute for Nuclear Research, Dubna, \\ Moscow Region, 114980, Russia and \\ Institute for Theoretical and Experimental Physics, Moscow, 117218, Russia \\ E-mail: kazakovdetheor.jinr.ru

\section{Şükrü Hanif Tanyıldızı ${ }^{* \dagger}$} \\ Bogoliubov Laboratory of Theoretical Physics, Joint Institute for Nuclear Research, Dubna, \\ Moscow Region, 114980, Russia \\ E-mail: hanifetheor.jinr.ru
}

\begin{abstract}
We study the cross-section of heavy Higgs production at the LHC within the framework of the Constrained MSSM. It is not only enhanced by $\tan ^{2} \beta$ but sometimes is also enhanced by the squark contribution. First, we consider the universal scenario within mSUGRA and find out that to get the desired enhancement one needs large negative values of $A_{0}$, which seems to be incompatible with the $b \rightarrow s \gamma$ decay rate. To improve the situation, we release the unification requirement in the Higgs sector. Then it becomes possible to satisfy all requirements simultaneously and enhance the squark contribution. The latter can gain a factor of several units increasing the overall cross-section which, however, is still smaller than the cross-section of the associated $H b \bar{b}$ production. We consider also some other consequences of the chosen benchmark point.
\end{abstract}

The XXth International Workshop High Energy Physics and Quantum Field Theory September 24 - October 1, 2011

Sochi, Russia

\footnotetext{
* Speaker.

${ }^{\dagger}$ This talk is based on arXiv:1106.4385.
} 


\section{Introduction}

Sometimes the heavy particle production can be enhanced by some factors as in the heavy Higgs production in the MSSM[1, 2]. For the case of gluon fusion it happens not only via $\tan \beta$ [3], but also via an additional source of enhancement due to the squark contribution in the loops. The Higgs production within the SM mainly goes through the gluon fusion process[4] (see Fig. 1). This is also true in the MSSM, though in this case the associated production with two b-quarks (two b-jets) is even more favorable[1]. The latter process is realized at the tree level and, hence, has no new virtual particles involved contrary to the loop diagrams. Nevertheless, the triangle diagrams do not give additional $b$-jets in the final states and can be distinguished from the associated production by b-tagging of these jets. At the same time, the squark contribution is also proportional to the quark mass, so only the third generation essentially plays any role. We proceed in two ways: First, we consider the usual MSSM universal high energy parameters $\left(m_{0}, m_{1 / 2}, A_{0}\right.$, and $\left.\tan \beta\right)$, evaluate masses and mixings, and calculate the cross-section for various points of parameter space. We find the areas in the parameter space where the loop enhancement takes place. This requires light topsquarks which is possible for very large and negative values of $A_{t}$ that implies negative $A_{0}$. Then we consider the fulfillment of various constraints such as $B \rightarrow X_{s} \gamma,[5] B_{s} \rightarrow \mu^{+} \mu^{-}$, [6, 7] $g-2$ of muon (see, e.g., Ref. [8]), relic density of the Dark Matter (DM)[9], electroweak precision data on $M_{W}$ and $\sin ^{2} \theta_{\text {eff }}$ (see, e.g., Ref. [10]), and Higgs and superpartner searches in this region. We find out that the considered universal scenarios with large negative $A_{0}$ are not compatible with the $b \rightarrow s \gamma$ constraint. To avoid this problem and to have the cross-section at the level of a few pb, we release the universality constraint and allow the non-universal Higgs mass (NUHM) terms[11]. As independent variables we take the Higgs mixing term $\mu$ and the CP-odd heavy Higgs boson mass $m_{A}$. We conclude the results in Sec.4.

\section{Cross-section for Heavy Higgs Production in the MSSM}

Our diagrams as in Fig. 1. Related gluon distribution functions is given by[1, 4, 12] and the
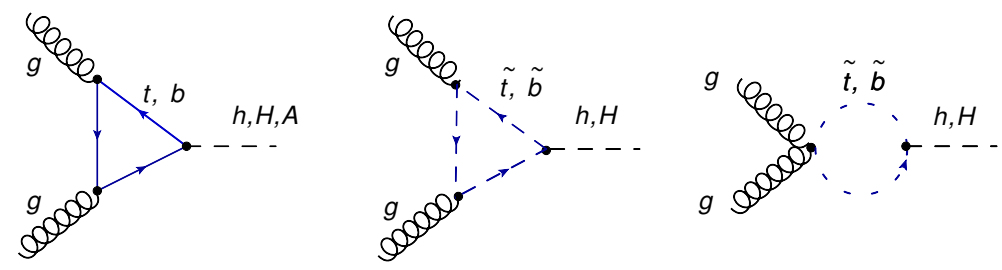

Figure 1: The leading order (LO) diagrams for the Higgs boson production via gluon fusion.

matrix elements by [13]. Due to the appearance of the quark mass squared versus $\tan \beta$, the main contribution comes from the t-squarks and not from the b-squarks. The triangle functions entering into the matrix elements, $F_{1 / 2}^{h, H, A}$ and $F_{0}$, are given by Ref. [14]. The triangle functions are complex functions of a single argument and get the imaginary part at the threshold when $m_{q_{i}}=m_{\text {Higgs }} / 2$, where the modulus of $F_{0}$ is maximal and saturates the squark contribution. Thus, the enhancement of the cross-section is achieved at the threshold when the mass of the t-squark and the heavy Higgs 
boson are correlated and differ by a factor of $1 / 2$. The values of quark masses and $\alpha_{s}$ should be taken at the $m_{\text {Higgs }}$ scale. In what follows all the needed low-energy running parameters are calculated with the help of SOFTSUSY 3.1.6[15] code which does not only perform the RG evolution but also incorporates the important threshold effects, in particular, to the b-quark mass[16], which is essential for our analysis.

\section{Non-universal soft supersymmetry breaking}

The non-universality in the Higgs sector parameterized by the pole mass of the CP-odd heavy boson $m_{A}$ and the running $\mu$-parameter at the SUSY scale (NUHM scenario) provides us with the possibility to overcome the difficulties of the universal scenarios for both negative and positive $A_{0}$. For the large $\tan \beta$ scenarios $m_{H} \simeq m_{A}$ and we have enough freedom to obtain significant enhancement in the $\sigma_{g g \rightarrow H}$ cross-section by adjusting $m_{A}$. Moreover, since we also can adjust the $\mu$ parameter, it is possible to fulfill the $b \rightarrow s \gamma$ constraint by the increase of the chargino contribution mentioned in the previous section. In the first place, we consider the case with negative $A_{0}$ and try to find a region that satisfies all the above-mentioned (see in Sec. 1) experimental constraints. In order to enhance the chargino contribution to the $b \rightarrow s \gamma$ decay rate, we need to decrease the value of the $\mu$ parameter. This, in turn, lowers the scale of stop masses. As a consequence, the lightest stop can become an LSP or even a tachyon if we consider very large values of $\left|A_{0}\right|$ which were chosen in the previous section. This kind of reasoning justifies our choice of $A_{0}$ given below. As a benchmark point we have used the following set of NUHM parameters $m_{1 / 2}=250 \mathrm{GeV}, m_{0}=625 \mathrm{GeV}$, $\mu=240 \mathrm{GeV}, m_{A}=340 \mathrm{GeV}, A_{0}=-1175 \mathrm{GeV}, \tan \beta=30$. This point lies in the region bounded by the experimental constraints mentioned above. Obviously, it is very hard to visualize the allowed region in the space of six free parameters. In what follows, we present in Fig. 2 the two-dimensional sections of the region in $m_{0}-A_{0}, m_{A}-\mu$ and $\tan \beta-A_{0}$ planes, respectively. One can see how the allowed regions due to various constraints intersect with each other. For the calculation of the flavour observables and the relic density we use the SuperIso (Relic) code[17, 18, 19], and the bounds for $b \rightarrow s \gamma^{1}, B_{s} \rightarrow \mu^{+} \mu^{-}$, and $\Omega h^{2}$ correspond to $95 \% \mathrm{CL}$. A point marked by the cross corresponds to the chosen benchmark scenario. This choice is somewhat random within the allowed region. Looking at the plot in Fig. 2 one can see where the allowed region moves when varying one or more parameters. For example, looking at the $A_{0}-\tan \beta$ plane one can deduce that with a slight increase of $\tan \beta$ both the $b \rightarrow s \gamma$ and the $B_{s} \rightarrow \mu^{+} \mu^{+}$rates go up and the allowed strips in the $m_{0}-A_{0}$ and $m_{A}-\mu$ planes effectively move towards the lower values in the corresponding figures. However, the correlations between the degrees of freedom are strong enough, so that it is hard to get the entire picture. In what follows we try, at least qualitatively, to explain the key features of the emerged picture. Since the stop mass scale depends crucially on the $m_{1 / 2}$ parameter, we restrict ourselves to the value $m_{1 / 2}=250 \mathrm{GeV}$. All the other parameters are allowed to vary. It turns out that the constraints due to the muon anomalous magnetic moment and electroweak precision data ${ }^{2}$ are satisfied in the whole region studied $\left(1 \lesssim a_{\mu} \times 10^{9} \lesssim 2.5, \Delta \rho \lesssim 5 \cdot 10^{-4}\right)$, so we do not draw the corresponding bounds. In the same figures, the SUSY enhancement of the Higgs production

\footnotetext{
${ }^{1}$ The allowed interval for $b \rightarrow s \gamma$ includes also theoretical uncertainties, see e.g. Ref. [17].

${ }^{2}$ We use FeynHiggs 2.7.4[20] to evaluate $\Delta \rho$ that parametrizes the leading universal corrections to the electroweak precision observables.
} 
via the gluon fusion is demonstrated with the help of the ratio $R_{H}=\sigma_{q+\tilde{q}} / \sigma_{q}$. Clearly, due to the fact that the quark contribution for our case is not very small, the enhancement is not very big in comparison with the results presented in the previous section, e.g., $R_{H} \sim 5$ for the benchmark point. Again, the value of $R_{H}$ correlates with $x_{\tilde{t}_{1}} \equiv 4 \tilde{m}_{t 1}^{2} / m_{H}^{2}$. At the lightest stop production threshold it is maximal and $R_{H}$ decreases more rapidly when $x_{\tilde{t}_{1}}>1$. In spite of the moderate enhancement the total cross-section is of the order of $\mathrm{pb}$ at the stop production threshold. At the top of Fig. 2 the plane $m_{0}-A_{0}$ is shown for fixed $\tan \beta=30, m_{1 / 2}=250 \mathrm{GeV}, m_{A}=340 \mathrm{GeV}$, and $\mu=250 \mathrm{GeV}$. One can see that the parameters $A_{0}$ and $m_{0}$ are correlated within the allowed band. This correlation corresponds to a constant value of the lightest stop mass lying in the range $150-200 \mathrm{GeV}$ and can be easily explained by the fact that an increase in the stop mass with $m_{0}$ can be compensated via a see-saw like mechanism by an increase in the off-diagonal term in the stop mass matrix driven by the absolute value of $A_{t}$. Clearly, both the $B \rightarrow \mu^{+} \mu^{-}$and $B \rightarrow X_{s} \gamma$ rates go down with $\tilde{m}_{t 1}$. In the middle of Fig. 2, we show how the allowed bands due to various constraints intersect in the $m_{A}-\mu$ plane. One can notice the dependence of the $b \rightarrow s \gamma$ rate on $\mu$ which somehow supports our hypothesis about the dominance of the chargino contribution to $C_{7}$ Wilson coefficient for small $\mu$. With the increase of $m_{A}$ the charged Higgs mass increases correspondingly. As a consequence, the sum $C_{7}^{H}+C_{7}^{\chi}$ becomes bigger, thus, slightly increasing the branching fraction. The correct amount of the Dark Matter can be achieved if LSP annihilates via the virtual CP-odd Higgs boson in the s-wave. For this to happen, the neutralino mass $m_{\chi^{0}}$ should be adjusted to half $m_{A}$. In our case, for fixed $m_{1 / 2}=250 \mathrm{GeV}$ the neutralino is mostly bino with $m_{\chi^{0}}$ around $100 \mathrm{GeV}$. Moreover, if $\mu$ is comparable with $m_{1 / 2}$ the fraction of higgsino component in $\chi^{0}$ becomes larger and also increase the cross-section which is proportional to the mixing between the gaugino and higgsino components for the s-wave annihilation. These two facts explain, at least qualitatively, the behavior of the curves with constant value of the DM relic density. For low $\mu \sim 200 \mathrm{GeV}$ it is sufficient to have $m_{A} \simeq 400 \mathrm{GeV}$ to obtain the correct value of $\Omega h^{2}$. However, when due to the increase of $\mu$ the mixing between the gaugino and higgsino components becomes small, one needs to lower $m_{A}$ to be closer to the $A_{0}$-resonance to enhance the annihilation cross-section. For the considered value of $\tan \beta=30$ the upper bound from $B_{s} \rightarrow \mu^{+} \mu^{-}$excludes $m_{A} \lesssim 330 \mathrm{GeV}$. All the constraints are satisfied in the small region near our benchmark point. Finally, at the bottom of Fig. 2 the $\tan \beta-A_{0}$ plane is shown. It is easy to notice that large $\tan \beta \gtrsim 30$ are excluded by the $B_{s} \rightarrow \mu^{+} \mu^{-}$ constraint since the dominant SUSY contribution to this decay scales as $\tan ^{6} \beta$.[21] In the allowed strip due to the $b \rightarrow s \gamma$ constraint the parameters $A_{0}$ and $\tan \beta$ are correlated since the enhancement due to $\tan \beta$ is compensated by the increase of $\tilde{m}_{t 1}$ due to $A_{0}$. The relic density constraint fixes $\tan \beta$ to be around 30. A tail of the $\Omega h^{2}$ region corresponds to the stop co-annihilation. In summary, the key features of the allowed region are the following: $m_{1 / 2} \sim \mu \sim 250 \mathrm{GeV}$ (which influence significantly the lightest stop mass, $b \rightarrow s \gamma$, and the mass and content of the lightest neutralino), $\tan \beta \sim 30$ (mostly due to the $\Omega h^{2}$ constraint), $m_{0}$ and $A_{0}$ should be correlated (due to the stop mass), and $m_{A} \gtrsim 300$ should not be very large (to have the Higgs production cross-section at the level of $1 \mathrm{pb})$. For our benchmark point the heavy CP-even Higgs boson decays predominatly into the heavy down-type fermions, i.e., $b \bar{b}(\sim 90 \%), \tau \bar{\tau}(\sim 10 \%)$. The latter signature has already been analyzed by both the ATLAS[22] and CMS[23] collaborations and important bounds on $m_{A}$ and $\tan \beta$ were deduced. However, the scenarios with $m_{A}>300 \mathrm{GeV}$ and $\tan \beta<50$ are not excluded at the moment. Before going to conclusions let us mention the situation with the case 

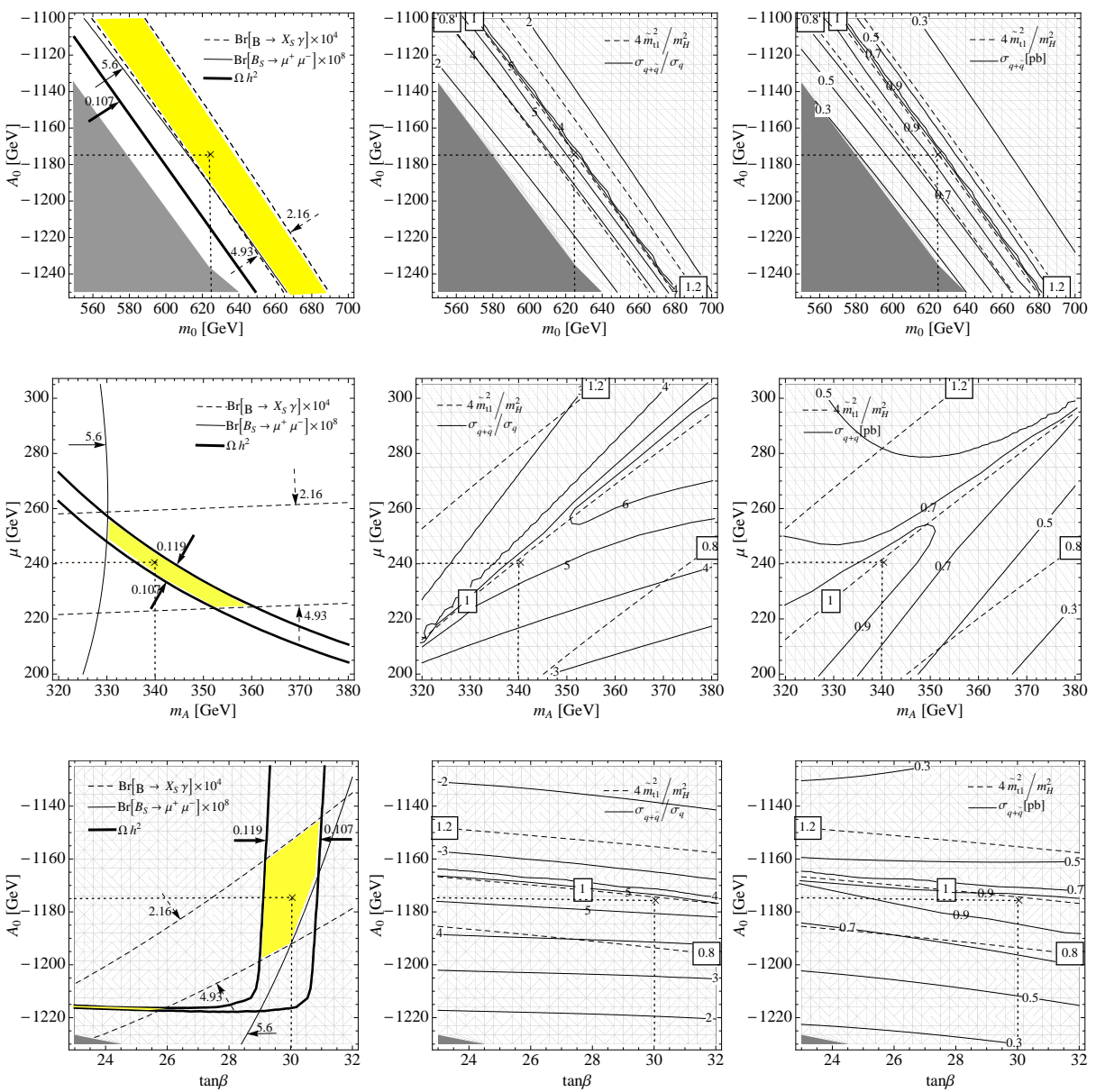

Figure 2: The allowed regions in the $m_{0}-A_{0}$ plane for $\tan \beta=30, m_{1 / 2}=250 \mathrm{GeV}, m_{A}=340 \mathrm{GeV}, \mu=240$ $\mathrm{GeV}$ (top), $m_{A}-\mu$ plane for $\tan \beta=30, m_{0}=625 \mathrm{GeV}, m_{1 / 2}=250 \mathrm{GeV}, A_{0}=-1175 \mathrm{GeV}$ (middle) and $\tan \beta-A_{0}$ plane for $m_{0}=625 \mathrm{GeV}, m_{1 / 2}=250 \mathrm{GeV}, m_{A}=340 \mathrm{GeV} \mu=240 \mathrm{GeV}$ (bottom). On the left panel, all of the regions between the arrows are allowed by the $B_{S} \rightarrow \mu^{+} \mu^{-}, B \rightarrow X_{S} \gamma$ and $\Omega h^{2}$ constraints. The intersection of these regions is marked by yellow. The ratio of the cross-sections $R_{H}=\sigma_{q+\tilde{q}} / \sigma_{q}$ and the total cross-section $\sigma_{q+\tilde{q}}$ at $\sqrt{s}=14 \mathrm{TeV}$ are shown on the middle and the right panels, respectively. The numbers $0.8,1.0$ and 1.2 on the middle and right panels correspond to the values of the ratio $4 \tilde{m}_{t 1}^{2} / \tilde{m}_{H}^{2}$. The benchmark point is marked by a cross.

of $A_{0}>0$. It should be noted that contrary to the $A_{0}<0$ case, positive $A_{0}$ leads to destructive interference between the squark and quark amplitudes at the stop threshold in the cross-section for heavy neutral Higgs production. The only possibility to enhance the cross-section is to be slightly below the threshold $\tilde{m}_{t 1} \lesssim m_{H} / 2$ when the corresponding squark amplitude develops a negative imaginary part. If we choose $m_{A}$ to be around $350-400 \mathrm{GeV}$, the SUSY enhancement with $R_{H} \sim 10$ is possible for $\tilde{m}_{t 1} \simeq 110 \mathrm{GeV}$. However, due to the behaviour of RGE for $A_{t}$, the large initial values of $A_{0}>0$ lead to a relatively small positive $A_{t}$ at the SUSY scale. In order to obtain the light stop needed for large $R_{H}$ via the see-saw like mechanism, the overall stop mass scale should not be very big. Unfortunately, this latter fact prevents us from finding a suitable region in the parameter space 
with $A_{0}>0$, since it turns out that for a setup like this the lightest Higgs boson mass is around $100 \mathrm{GeV}$, which is excluded experimentally (we use HiggsBounds 2.0[24] package for confronting our predictions with the LEP bound). In contrast, for the $A_{0}<0$ scenario we have $m_{h_{0}} \simeq 118 \mathrm{GeV}$.

\section{Discussion}

The search for the Higgs boson seems to be the main goal for the LHC today though the appearance of the new physics would be the major breakthrough. One can see that even if the "new physics" is represented by the enlargement of the Higgs sector, the cross-section of the Higgs production can be essentially enhanced due to the large value of $\tan \beta=v_{2} / v_{1}$. This enhancement might even lead to preferable observation of a heavy Higgs boson rather than the light one. At the same time, if SUSY or some other heavy particles exist, the enhancement of the Higgs production can be pushed even further. This latter enhancement, however, is valid only for the restricted set of parameters subjected to two requirements: one of the intermediate particles (the lightest top squark $\tilde{t}_{1}$ in our case) has to be relatively light and has to be close to the resonance with the Higgs boson. The allowed region in the parameter space found here seems to be very narrow mostly due to the relic density constraint. However, this impression is not true since in each plane shown in Fig. 2 all the other parameters are fixed. In the whole parameter space the allowed volume with $\sigma_{q+\tilde{q}} \lesssim 1$ $\mathrm{pb}$ and $R_{H} \simeq 3-5$ is obviously bigger. Our main goal was to study the influence of squarks on the heavy Higgs boson production and to find the regions of the MSSM parameter space, for which the cross-section via the gluon fusion process can be essentially increased. However, in the considered scenarios compatible with known experimental constraints it is still lower than the associated production accompanied by two b-quarks[1]. Indeed, with the help of CalcHep[25] package the total cross-section for $p p \rightarrow \bar{b} b H$ process is estimated to be around $7 \mathrm{pb}$ at $\sqrt{s}=14 \mathrm{TeV}^{3}$ for our benchmark point $\left(\tan \beta=30\right.$, and $\left.M_{A}=340 \mathrm{GeV}\right)$. This is an order of magnitude larger than the gluon fusion cross-section evaluated above. Hence, it is very hard to "see" the gluon-fusion on top of the $b \bar{b} H$ process in which there are no virtual superpartners, so the same cross-section is expected within any Two-Higgs Doublet Model (THDM) with large $\tan \beta$. As a conseqence, a complimentary search is required to discriminate between different THDM possibilities. It is worth mentioning the other phenomenological implications of the chosen benchmark point with $A_{0}<0$. In the considered case the lightest top squark is almost degenerate with the top quark and its dominant decay channel is $\tilde{t}_{1} \rightarrow \chi_{1}^{+} b$ (we use SUSYHIT code[27] to calculate the branching fractions). This mode was not so extensively analyzed at the Tevatron and the current bounds for the stop production at $\sqrt{s}=1.96 \mathrm{TeV}$ are far above the theoretically predicted values[28]. However, at the LHC they can be produced abundantly. For example, for our benchmark point the stop pair production cross-section at $\sqrt{s}=14 \mathrm{TeV}$ that was obtained with the help of the Calchep package[25] is around $55 \mathrm{pb}$ (in comparison with approximately $8 \mathrm{pb}$ for $\sqrt{s}=7 \mathrm{TeV}$ ). The lightest chargino $\chi_{1}^{+}$produced in the stop decay has the mass slightly below the neutralino-W-boson threshold $\left(m_{\chi^{+}} \simeq 170 \mathrm{GeV} \lesssim m_{\chi^{0}}+m_{W}\right)$, so it decays into the lightest neutralino and a fermion-antifermion pair coming from the virtual $W$-boson. It turns out that the chargino decays into the light quarks with $66 \%$ probability. In $33 \%$ cases it produces leptons. As a consequence, we have the

\footnotetext{
${ }^{3}$ Comparison is made with bbh@nnlo package[26] and a reasonable agreement is found.
} 
following key signature for the stop pair production: two b-jets coming from the decay of the stops, missing energy $\not_{T}$ from two neutralinos, and light-quark jets or leptons from the virtual $W$-bosons (see Fig. 3). It is obvious that for the considered value of the stop mass the final states are similar to that of the top pair production so one can search for $\tilde{t}_{1} \overline{\tilde{t}}_{1}$ signal in the $t \bar{t}$ event sample as it was done in Ref. [28]. The ATLAS collaboration has already performed a study of such signatures[29]

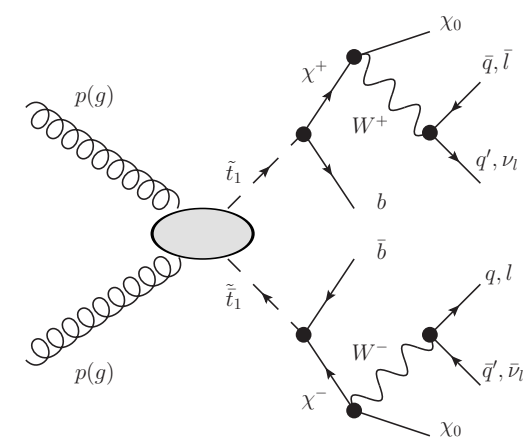

Figure 3: The lightest stop pair-production process at the LHC energies in proton-proton collisions. The blob corresponds to all the tree-level diagrams contributing to the stop production. The final states include two b-jets, missing energy $\phi_{T}$, the light-quark jets, and leptons. With almost equal probability (45\%) the virtual $W$-boson produces either four jets or two jets accompanied by a charged lepton and additional missing energy from neutrino. In $10 \%$ of cases two $W$-bosons decay leptonically, and instead of the light-quark jets we have two charged leptons and additional $\phi_{T}$ from two neutrinos.

at $\sqrt{s}=7 \mathrm{TeV}$ with real data obtained in 2010 (so-called one-lepton analysis with b-jets and missing transverse energy). Their results can be interpreted as exclusion limits in the $\left(m_{\tilde{g}}, \tilde{m}_{t 1}\right)$ plane ( $m_{\tilde{g}}$ being the gluino mass) and, according to Fig. 3 of Ref. [29], the stop production cross-section should be smaller than $15-40 \mathrm{pb}$ for $\tilde{m}_{t 1} \simeq 180 \mathrm{GeV}$ depending on the gluino mass which varies in the range $350-620 \mathrm{GeV}$. Since for our benchmark point the cross-section of stop pair production with the given final states is approximately $\sigma_{\tilde{t}_{1}} \overline{\tilde{t}}_{1} \times \mathrm{BR}\left(\tilde{t}_{1} \overline{\tilde{t}}_{1} \rightarrow b q q^{\prime} b l v\right)=8 \times 0.45=3.6 \mathrm{pb}$ and $m_{\tilde{g}} \simeq 630 \mathrm{GeV}$, it seems that we escape the current ATLAS bound. However, the searches for the light stop production seems to be very challenging and we attract attention to this decay mode. Another interesting point is that the $B_{s} \rightarrow \mu^{+} \mu^{-}$branching fraction almost touches the experimentally allowed boundary line so it may happen that this rare decay would be observed at the LHCb experiment in the near future. Thus, our main conclusion is that there exists a possibility when the cross-section of the single Higgs production is large enough to favour its observation at the LHC even with intermediate luminosity. In addition, the search for the lightest stop production in the $\tilde{t} \rightarrow \chi^{+} b$ mode seems to be within the reach of the LHC at the early stage. Whether we are lucky or not will be clear only a posteriori. However, any favourable possibility should not be missed.

\section{References}

[1] A. Djouadi, Phys. Rep. 459, 1 (2008) hep-ph/0503173.

[2] LHC Higgs Cross Section Working Group Collaboration (S. Dittmaier et al.), Handbook of LHC Higgs Cross Sections: 1. Inclusive Observables, arXiv:1101.0593 [hep-ph]. 
[3] C. Beskidt, W. de Boer, T. Hanisch, E. Ziebarth, V. Zhukov, D.I. Kazakov, Phys. Lett. B 695, 143 (2011), arXiv:1008.2150 [hep-ph].

[4] H. M. Georgi, S. L. Glashow, M. E. Machacek et al., Phys. Rev. Lett. 40, 692 (1978).

[5] Heavy Flavor Averaging Group (E. Barberio et al.), Averages of $b$-hadron and $c$-hadron Properties at the End of 2007, arXiv:0808.1297 [hep-ex].

[6] CDF Collaboration (T. Aaltonen et al.), Phys. Rev. Lett. 100, 101802 (2008), arXiv:0712.1708 [hep-ex].

[7] D0 Collaboration (V. M. Abazov et al.), Phys. Lett. B 693 , 539 (2010), arXiv:1006.3469 [hep-ex].

[8] D. Stockinger, J. Phys. G 34, R45 (2007), arXiv:hep-ph/0609168].

[9] WMAP Collaboration (E. Komatsu et al.), Astrophys. J. Suppl. 180 , 330 (2009), arXiv:0803.0547 [astro-ph].

[10] S. Heinemeyer, W. Hollik, G. Weiglein, Phys. Rept. 425, 265 (2006), hep-ph/0412214.

[11] J. R. Ellis, K. A. Olive, Y. Santoso, Phys. Lett. B 539 , 107 (2002), hep-ph/0204192.

[12] M. Spira, A. Djouadi, D. Graudenz, P.M. Zerwas, Phys. Lett. B 318 , 347 (1993).

[13] J. F. Gunion, H. Haber, G. Kane and S. Dawson, The Higgs HunterâĂŹs Guide, (Addison- Wesley Publishing Company, Redwood City, CA, 1990).

[14] L. B. Okun, Leptons and Quarks, (Elsevier Science Pub Co, March 1, 1985).

[15] B. C. Allanach, Comput. Phys. Commun. 143, 305 (2002), hep-ph/0104145.

[16] M. S. Carena, D. Garcia, U. Nierste, C. E. M. Wagner, Nucl. Phys. B 577, 88 (2000), hep-ph/9912516.

[17] F. Mahmoudi, Comput. Phys. Commun. 180, 1579 (2009), arXiv:0808.3144 [hep-ph].

[18] F. Mahmoudi, Comput. Phys. Commun. 180, 1718 (2009)

[19] A. Arbey, F. Mahmoudi, Comput. Phys. Commun. 181, 1277 (2010), arXiv:0906.0369 [hep-ph].

[20] T. Hahn, S. Heinemeyer, W. Hollik, H. Rzehak, G. Weiglein, Nucl. Phys. Proc. Suppl. 205-206, 152 (2010), arXiv:1007.0956 [hep-ph].

[21] C. Bobeth, T. Ewerth, F. Kruger, J. Urban, Phys. Rev. D 64, 074014 (2001), hep-ph/0104284.

[22] ATLAS Collaboration (M. Schumacher), Higgs Boson Searches with ATLAS based on 2010 Data, arXiv:1106.2496 [hep-ex].

[23] CMS Collaboration (S. Chatrchyan et al.), Search for Neutral MSSM Higgs Bosons Decaying to Tau Pairs in $p p$ Collisions at $\sqrt{s}=7 \mathrm{TeV}$, arXiv:1104.1619 [hep-ex].

[24] P. Bechtle, O. Brein, S. Heinemeyer, G. Weiglein and K. E. Williams, HiggsBounds 2.0.0: Confronting Neutral and Charged Higgs Sector Predictions with Exclusion Bounds from LEP and the Tevatron, arXiv:1102.1898 [hep-ph].

[25] A. Pukhov, Calchep 2.3: MSSM, structure functions, event generation, and generation of matrix elements for other packages, arXiv:hep-ph/0412191.

[26] R. V. Harlander and W. B. Kilgore, Phys. Rev. D 68, 013001 (2003), arXiv:hep-ph/0304035.

[27] A. Djouadi, M. M. Muhlleitner, M. Spira, Acta Phys. Polon. B 38, 635 (2007), hep-ph/0609292.

[28] DO Collaboration (V. M. Abazov et al.), Phys. Lett. B 674, 4 (2009). arXiv:0901.1063 [hep-ex].

[29] ATLAS Collaboration (G. Aad et al.), Search for supersymmetry in pp collisions at $\sqrt{s}=7 \mathrm{TeV}$ in final states with missing transverse momentum and b-jets, arXiv:1103.4344 [hep-ex]. 